\title{
Analysis of nonuniform and dispersive time domain reflectometry measurement systems with application to the dielectric spectroscopy of soils
}

\author{
C.-P. Lin \\ Department of Civil Engineering, National Chiao Tung University, Hsinchu, Taiwan
}

Received 1 May 2002; revised 14 October 2002; accepted 14 October 2002; published 18 January 2003.

[1] A practical time domain reflectometry (TDR) measurement system consists of a cable tester and a nonuniform transmission line. The transmission line includes a coaxial cable, a transitional device, and a measurement probe. Dielectric spectroscopy in a nonuniform transmission line requires a wave propagation model that accounts for multiple reflections in a nonuniform and dispersive TDR measurement system. Spectral analysis and the concept of input impedance were utilized to develop such a model. The system parameters were calibrated using the measured TDR waveform in an aqueous material with known dielectric permittivity. The excellent match between the simulated and measured waveforms validated the wave propagation model. A layer-peeling algorithm was developed, based on the new formulation of the wave propagation model to remove the effect of the cable and transitional device on the measurement by TDR. The dielectric spectrum obtained by solving the scatter function was compared with that obtained by matching waveforms based on a dielectric relaxation model. The latter method was confirmed by the experimental results as being superior to the former at high frequencies if an appropriate dielectric relaxation model was used. Both the Debye relaxation model and the volumetric mixing model could model the dielectric dispersion of soils in the TDR frequency range. The latter method outperforms the former in modeling the dielectric dispersion of soils over the full range of frequencies. INDEX TERMS: 1866 Hydrology: Soil moisture; 1894 Hydrology: Instruments and techniques; KEYWORDS: TDR, time domain reflectometry, transmission lines, dielectric spectroscopy, soil physics, soil moisture

Citation: Lin, C.-P., Analysis of nonuniform and dispersive time domain reflectometry measurement systems with application to the dielectric spectroscopy of soils, Water Resour. Res., 39(1), 1012, doi:10.1029/2002WR001418, 2003.

\section{Introduction}

[2] Time domain reflectometry (TDR) waveforms are practically analyzed by travel time analysis, from which the apparent dielectric constant can be determined. Heimovaara [1994] showed that the apparent dielectric constant was correlated with the real part of the dielectric permittivity at high frequencies $(\sim 1-2 \mathrm{GHz})$. In this frequency range the soil dielectric permittivity is not sensitive to the effects of soil's microstructure and soil-water interaction. Consequently, the apparent dielectric constant can be applied to measuring the volumetric water content of soils. In contrast, the spectrum of the soil dielectric permittivity at lower frequencies is useful not only for enhancing the accuracy of electromagnetic-based exploration methods, but also in providing valuable information about solid-liquid interactions. Earlier approaches to determine the frequencydependent dielectric properties of materials [Giese and Tiemann, 1975; Heimovaara, 1994] have used a sophisticated small cell or probe that is not appropriate for field applications or soils with large particles. A practical system for dielectric spectroscopy requires new development.

Copyright 2003 by the American Geophysical Union. 0043-1397/03/2002WR001418
[3] A typical TDR system consists of a cable tester and a nonuniform transmission line. The line comprises a coaxial cable, a transitional device (or probe head), and a measurement probe. Dielectric spectroscopy using a nonuniform transmission line requires a wave propagation model that accounts for multiple reflections in a nonuniform and dispersive TDR measurement system. If the cross-sectional dimensions of the line or the properties of the surrounding medium vary along the axis of the line, then the per-unitlength parameters are functions of the position variable $z$. This fact makes the resulting transmission-line equations more difficult to solve. Yanuka et al. [1988] described a model that accommodates multiple reflections in a nonuniform transmission line but does not consider the dependency of the material's dielectric permittivity on frequency. Heimovaara [1994] employed spectral analysis to account for dielectric dispersion, but his method considered only a uniform transmission line (that is, a matched system). Feng et al. [1999] combined the works of Yanuka [1988] and Heimovaara [1994] and formulated the wave propagation as a feedback linear-time-invariant (LTI) system. The LTI system successfully simulates TDR waveforms of nonuniform transmission lines with dispersive dielectric materials.

[4] This study presents a different approach to formulating multiple reflections in a nonuniform and dispersive 


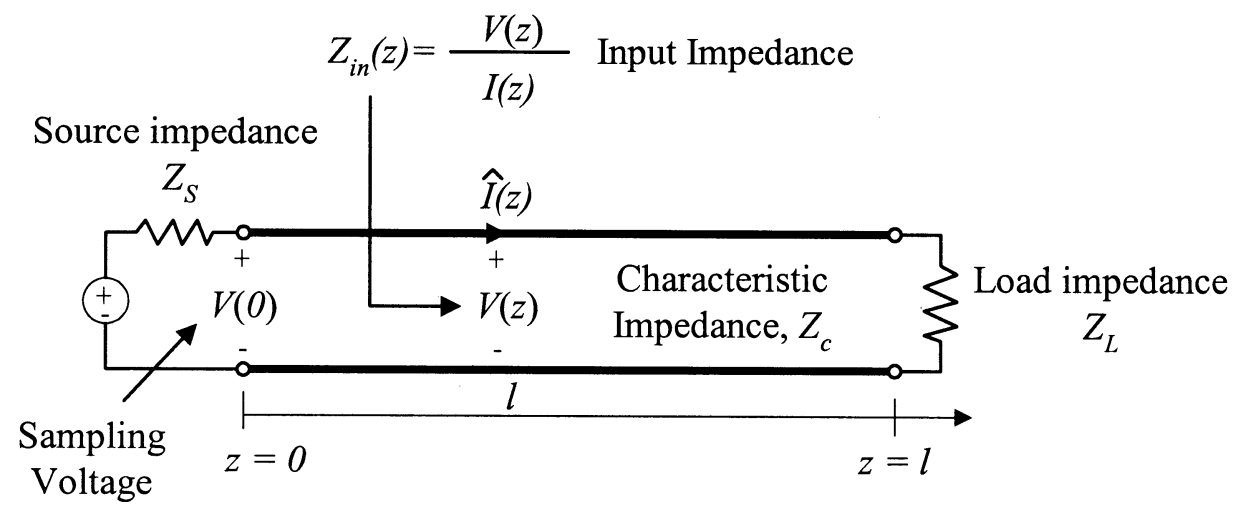

Figure 1. Equivalent circuit of a time domain reflectometry (TDR) system and definition of input impedance $Z_{\text {in }}$.

TDR measurement system using spectral analysis and the concept of input impedance. The new formulation is simpler and more systematic than that described by Feng et al. [1999], leading to a layer-peeling algorithm that removes the effect of the cable and transitional device on the TDR measurement. A relatively simple and robust measurement system is used to demonstrate the capability of the wave propagation model and layer-peeling algorithm for measuring dielectric spectrum.

\section{Theoretical Background}

\subsection{Transmission Line Theory}

[5] The line current $I$ and the voltage between the conductors $V$ in a transmission line can be uniquely defined to describe the electromagnetic wave propagation because of the special field structure (i.e., transverse electromagnetic model) inside the transmission line. The general solution for a uniform transmission line in terms of $V$ and $I$ in phasor forms is [Ramo et al., 1994]

$$
\begin{aligned}
& V(z)=V^{+} e^{-\gamma z}+V^{-} e^{\gamma z} \\
& I(z)=\frac{V^{+}}{Z_{c}} e^{-\gamma z}-\frac{V^{-}}{Z_{c}} e^{\gamma z}
\end{aligned}
$$

where $z$ is the position along the line, $V^{+}$and $V^{-}$are the two unknown constants in the general solution, $\gamma$ is the propagation constant (which is a function of dielectric permittivity), and $Z_{c}$ is the characteristic impedance (which is a function of dielectric permittivity and the crosssectional geometry of the transmission line). The terms $\gamma$ and $Z_{c}$ can be written as

$$
\begin{aligned}
& \gamma=\frac{j 2 \pi f}{c} \sqrt{\varepsilon^{*}} \\
& Z_{c}=Z_{p} / \sqrt{\varepsilon^{*}}
\end{aligned}
$$

where $j=(-1)^{1 / 2}, c$ is the speed of light, $\varepsilon^{*}$ is the complex dielectric permittivity, and $Z_{p}$ is the reference characteristic impedance, which is defined as the characteristic impedance of the transmission line filled with air. Notably, $Z_{p}$ is a function only of the cross-sectional geometry of the transmission line. Its value may be derived from electromagnetic theory or calibrated using material of known dielectric permittivity.

[6] In the case of a uniform transmission line, an equivalent circuit, as shown in Figure 1, can represent a TDR system. The boundary conditions are

$$
\begin{gathered}
V(0)=V_{S}-Z_{S} I(0) \\
V(l)=Z_{L} I(l)
\end{gathered}
$$

where $V_{S}$ represents the independent voltage source, $Z_{S}$ is the source impedance at $z=0$ (typically $Z_{S}=50 \Omega$ ), and $Z_{L}$ is the load impedance at $z=l\left(Z_{L}=\infty\right.$ for an open end or zero for a shorted end). The two boundary conditions can be applied to solving for the two unknown coefficients $V^{+}$and $V^{-}$. The voltage and current along the transmission line can then be obtained by substituting the solutions of $V^{+}$and $V^{-}$ back into equation (1). The solution of particular interest is for the voltage at $z=0$, which is the sampling voltage shown on the TDR oscilloscope.

[7] Alternatively, the input impedance approach may be used to extend the usefulness of the solution. The input impedance $Z_{i n}(z)$ is the equivalent impedance when looking into the circuit at position $z$ [Magnusson et al., 1992], as shown in Figure 1. The impedance at $z=0$ can be derived as a function of the input impedance at $z=l$ as

$$
Z_{\text {in }}(0)=Z_{c} \frac{Z_{L}+Z_{c} \tanh (\gamma l)}{Z_{c}+Z_{\text {in }}(l) \tanh (\gamma l)}
$$

[8] Using the terminal condition at $z=0$ and $I(0)=V(0) /$ $Z_{\text {in }}(0)$, the sampling voltage $V(0)$ can be obtained as

$$
V(0)=\frac{Z_{\text {in }}(0)}{Z_{\text {in }}(0)+Z_{S}} V_{S}
$$

\subsection{Dielectric Dispersion Models}

[9] The propagation constant and characteristic impedance in equation (1) are functions of the dielectric permittivity of the material in the transmission line. For a 
Table 1. Parameters of the Four-Component Mixing Model

\begin{tabular}{|c|c|c|c|c|c|c|c|c|c|c|c|c|c|c|c|c|}
\hline & \multicolumn{6}{|c|}{ Soil Matrix Parameters } & \multirow[b]{2}{*}{ Air, $\varepsilon_{a}^{*}$} & \multirow[b]{2}{*}{ Solid, $\varepsilon_{s}^{*}$} & \multicolumn{4}{|c|}{$\begin{array}{l}\text { Free Water } \varepsilon_{f}^{*} \\
\text { (Debye Model) }\end{array}$} & \multicolumn{4}{|c|}{$\begin{array}{c}\text { Bound Water } \varepsilon_{b}^{*} \\
\text { (Debye Model) }\end{array}$} \\
\hline & $\rho_{s}$ & $\theta$ & $\rho_{d}$ & $A_{e s}$ & $\delta$ & $\alpha$ & & & $\varepsilon_{d c}$ & $\varepsilon_{\infty}$ & $f_{\text {rel }}$ & $\sigma_{f w}$ & $\varepsilon_{d c}$ & $\varepsilon_{\infty}$ & $f_{\text {rel }}$ & $\sigma_{f w}$ \\
\hline Unit & $\mathrm{g} / \mathrm{cm}^{3}$ & $\%$ & $\mathrm{~g} / \mathrm{cm}^{3}$ & $\mathrm{~m}^{2} / \mathrm{g}$ & $\mathrm{m}$ & & & & & & $\mathrm{GHz}$ & $\mathrm{S} / \mathrm{m}$ & & & $\mathrm{kHz}$ & $\mathrm{S} / \mathrm{m}$ \\
\hline Assumed Value & 2.65 & $\mathrm{U}^{\mathrm{a}}$ & U & $\mathrm{U}$ & $3 \times 10^{-10}$ & $\mathrm{U}$ & 1 & 4.7 & 80 & 4.22 & 17.4 & $\mathrm{U}$ & 80 & 4.2 & 9 & $\mathrm{U}$ \\
\hline
\end{tabular}

${ }^{\mathrm{a} U n k n o w n}$

homogeneous material the frequency-dependent dielectric permittivity within the TDR bandwidth may be described by the Debye equation [Hasted, 1973]:

$$
\varepsilon^{*}(f)=\left[\varepsilon_{\infty}+\frac{\varepsilon_{d c}-\varepsilon_{\infty}}{1+f / f_{\text {rel }}}\right]-\frac{j \sigma}{2 \pi f \varepsilon_{0}}
$$

where $\varepsilon_{d c}$ is the static dielectric permittivity, $\varepsilon_{\infty}$ is the dielectric permittivity at an infinite frequency, $f_{\text {rel }}$ is the relaxation frequency, and $\sigma$ is the electrical conductivity of the material. The Debye equation can describe the dielectric property of each soil phase. This property is almost independent of frequency below microwave frequencies. The heterogeneity of a soil, however, adds to the complexity of its dielectric property and has three major effects: polarization of bound water, the Maxwell-Wagner effect, and polarization of the double layer [Hilhorst, 1998]. These interfacial effects cause apparent dielectric relaxation within the TDR bandwidth.

[10] The Debye equation has been used to model the apparent dielectric relaxation of soils, response functions, and time domain signals of the measurement system [Heimovaara et al., 1996; Friel and Or, 1999]. This approach uses a single relaxation to approximately capture the dielectric dispersion due to interfacial effects. A volumetric mixing model that sums two or more dielectric spectra with different Debye parameters may describe the dielectric dispersion of soils more realistically. Dobson et al. [1985] and Heimovaara et al. [1994] presented a four-component mixing model to predict the frequency-dependent complex permittivities of soils. It can be rewritten in terms of soil physical parameters as

$$
\begin{aligned}
\varepsilon_{m}^{* \alpha}= & \left(\frac{\rho_{d}}{\rho_{s}}\right) \varepsilon_{s}^{* \alpha}+\left(\theta-\delta \rho_{d} A_{s}\right) \varepsilon_{f w}^{* \alpha}+\left(\delta \rho_{d} A_{s}\right) \varepsilon_{b w}^{* \alpha} \\
& +\left(1-\frac{\rho_{d}}{\rho_{s}}-\theta\right) \varepsilon_{a}^{* \alpha}
\end{aligned}
$$

where $\varepsilon_{s}^{*}, \varepsilon_{f w}^{*}, \varepsilon_{b w}^{*}$, and $\varepsilon_{a}^{*}$ are the complex dielectric permittivity of soil solids, free water, bound water, and air, respectively; $\alpha$ is a fitting parameter; $\rho_{d}$ is the soil's bulk dry density; $\rho_{s}$ is the mean density of the solid phase; the product $\delta \rho_{d} A_{s}$ represents the volumetric bound water content; $\delta$ is the mean thickness of the bound water; and $A_{s}$ is the specific surface area of the soil. The complex dielectric permittivity of each phase is either constant or given by the Debye equation. Parameters that do not vary greatly are assumed to be constant and represented by their typical values in Table 1 , where $\sigma_{f w}$ and $\sigma_{b w}$ are the conductivity of free water and bound water, respectively. Accordingly, the volumetric mixing equation includes six unknown parameters, namely, $\theta, \rho_{d}, A_{s}, \sigma_{f w}, \sigma_{b w}$, and $\alpha$, to describe the dielectric dispersion of soils.

\section{Methods}

\subsection{TDR Measurements}

[11] TDR measurements were made using a Tektronix 1502B TDR cable tester connected to a TDR probe via a 3 -foot Intcom $50-\Omega$ cable. The rise time of the step pulse was about $200 \mathrm{ps}$, corresponding to a bandwidth of $1.5 \mathrm{GHz}$ (TDR bandwidth $\approx 1 /(\pi \times$ rise time $)$ ). The horizontal scale and velocity of propagation $\left(V_{p}\right)$ were set here to $0.1 \mathrm{~m} /$ division and 0.99 , respectively. The sampling frequency at this scale was $37.1 \mathrm{GHz}$, well above double the TDR frequency bandwidth. The TDR probe was a coaxial cylinder with a central rod, as shown in Figure 2. The diameter of the central rod and the inside diameter of the cylinder were 8 and $102 \mathrm{~mm}$, respectively. The height of the cylinder mold was $116 \mathrm{~mm}$. A probe head provided the necessary transition from the coaxial cable to the coaxial probe. It was comprised of three components: (1) a 50- $\Omega$ mating BNC connector; (2) a metal cylindrical head with Delrin $\left.{ }^{(}\right)$as the insulating material, and (3) a multiple rod section that consisted of a central rod and three perimeter conducting rods with air as the insulating material. The configuration depicted in Figure 2 was used to perform laboratory experiments in this study. Similarly, field measurements can be made using the same probe head and four parallel metal rods. This type of probe has been frequently used to measure water content; however,

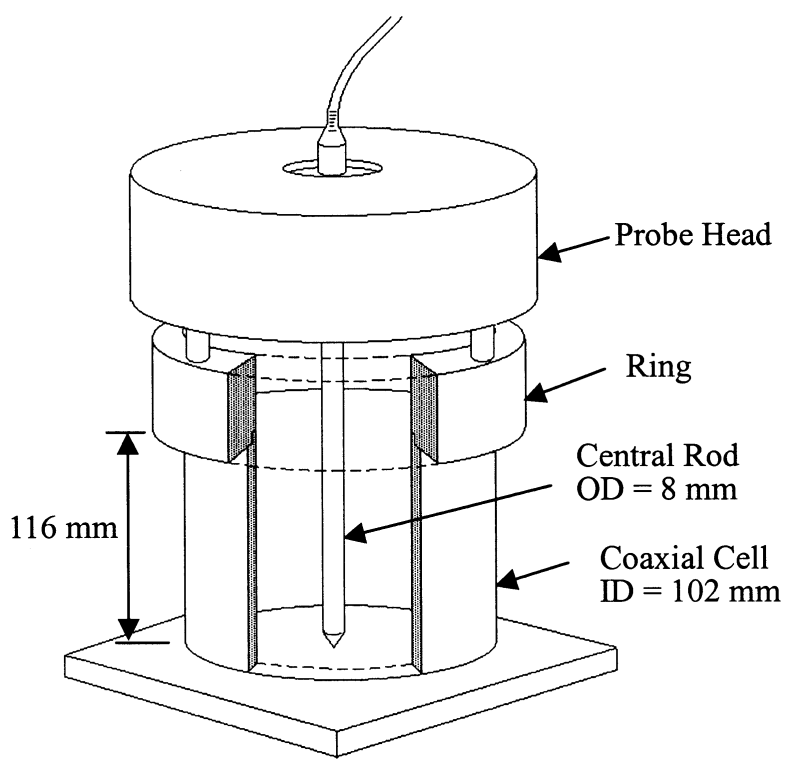

Figure 2. Coaxial waveguide used in this study. 


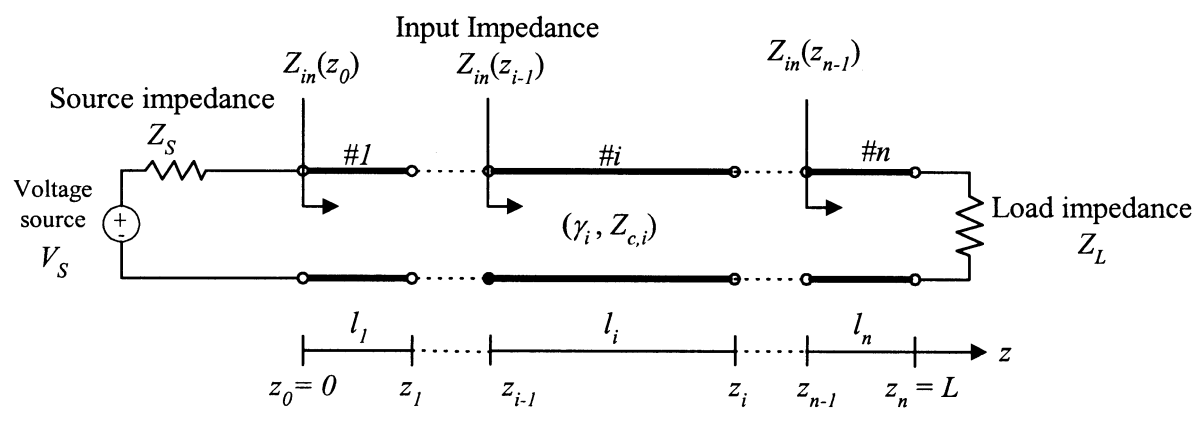

Figure 3. Representing a nonuniform line as a cascade of uniform sections.

it is not suited to dielectric spectroscopy. Methods are developed here to solve this problem.

[12] TDR waveforms of deionized water, tap water, ethanol alcohol, and butanol alcohol were measured to calibrate and validate the methods developed. Measurements were also taken on a compacted soil to test the applicability of the new method to soils. The soil, composed of $20 \%$ sand, $55 \%$ silt, and $25 \%$ clay, was fist mixed with some water to the desired water content. After the soil was placed and compacted in the cylinder mold, the total weight of the soil and mold was obtained. A central rod was then installed and the TDR measurement made. Then, the soil was oven-dried to determine the water content.

\subsection{Scatter Function of a Nonuniform Transmission Line}

[13] The above TDR system includes a cable tester and a nonuniform transmission line. The resulting transmission line equations became nonconstant-coefficient differential equations. However, a cascade of uniform sections, as shown in Figure 3, could be used to discretize the nonuniform transmission line. Hence equation (1) still describes the general solution for each of the $n$ uniform sections. Therefore a total of $2 n$ unknown coefficients $\left(V_{i}^{+}, V_{i}^{-}\right)$are involved. The terminal conditions are the same as in equation (3), leaving $2 n$ unknown coefficients and only two boundary conditions. The continuity constraints at the discontinuities between the terminations yield $2(n-1)$ more equations; hence the $2 n$ unknown coefficients could be determined from the $2 n$ constraints. Rather than solving the simultaneous equations, a more systematic and explicit procedure involves calculating the input impedance from the end to the source. The analysis starts by determining the input impedance at the end of the line, transforming the impedance successively to the subsequent discontinuity until the source is reached. This analysis is performed using equation (4) in a bottom-up fashion, relating the impedance at the two ends of the uniform section of transmission line:

$$
\begin{gathered}
Z_{i n}\left(z_{n}\right)=Z_{L} \\
Z_{\text {in }}\left(Z_{n-1}\right)=Z_{c, n} \frac{Z_{L}+Z_{c, n} \tanh \left(\gamma_{n} l_{n}\right)}{Z_{c, n}+Z_{L} \tanh \left(\gamma_{n} l_{n}\right)} \\
Z_{\text {in }}\left(z_{n-2}\right)=Z_{c, n-1} \frac{Z_{\text {in }}\left(z_{n-1}\right)+Z_{c, n-1} \tanh \left(\gamma_{n-1} l_{n-1}\right)}{Z_{c, n-1}+Z_{\text {in }}\left(z_{n-1}\right) \tanh \left(\gamma_{n-1} l_{n-1}\right)} \\
\vdots \\
Z_{\text {in }}(0)=Z_{c, l} \frac{Z_{\text {in }}\left(z_{1}\right)+Z_{c, 1} \tanh \left(\gamma_{1} l_{1}\right)}{Z_{c, 1}+Z_{\text {in }}\left(z_{1}\right) \tanh \left(\gamma_{1} l_{1}\right)}
\end{gathered}
$$

where $Z_{c, i}, \gamma_{i}$, and $l_{i}$ are the characteristic impedance, propagation constant, and length of each section, respectively. Once the input impedance looking into the entire line is obtained from equation (8), the sampling voltage $V(0)$ can then be determined from equation (5).

[14] The voltage source in equation (5) is a step pulse with a rise time of $200 \mathrm{ps}$, according to the specifications of the Tektronix 1502B. However, its exact and complete waveform is unknown. The incident waveform of the TDR system can be experimentally obtained by terminating the cable tester with a $50-\Omega$ impedance block (such that no reflection occurs). Substituting $Z_{\text {in }}(0)=50 \Omega$ into equation (5) yields the measured waveform equal to the incident waveform $\left(V_{\text {in }}\right)$. Thus

$$
V_{\text {in }}=\frac{V_{S}}{2}
$$

[15] The solution of the sampling voltage in equation (5) can be rewritten in terms of an incident wave and a reflected wave from the transmission line as

$$
V(0)=V_{\text {in }}+S_{11} V_{\text {in }}
$$

in which the scatter function $S_{11}$ is defined as

$$
S_{11}=\frac{Z_{\text {in }}(0)-Z_{S}}{Z_{\text {in }}(0)+Z_{S}}
$$

\subsection{Modeling TDR Waveforms}

[16] The scatter function derived above can be used to simulate TDR waveforms for any TDR measurement system with various types of transmission lines and insulating materials. The waveform of the incident wave from the Tektronix 1502B into the transmission line was determined by terminating the cable test device with a $50-\Omega$ impedance block, as shown in Figure 4. The bumps following the rising step were recognized to be aberrations due to the internal circuitry and reflections from the front panel. These bumps are not part of the incident step pulse. The measured waveform was modified to eliminate the bumps, as shown in Figure 4. Evaluating the overall scatter function for a given TDR measurement system requires that the length $l_{i}$, the reference impedance $Z_{p i}$, the dielectric permittivity $\varepsilon_{i}^{*}$ of each uniform section of the nonuniform transmission line, 


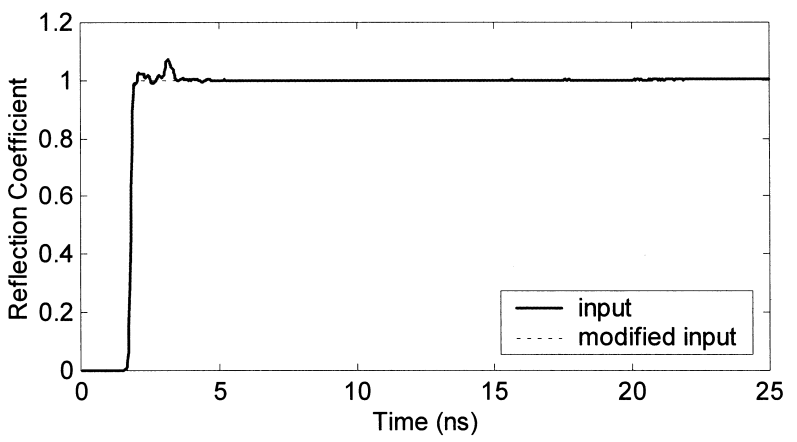

Figure 4. TDR step input.

and the terminal impedances, $Z_{S}$ and $Z_{L}$, all be known. After all these line parameters were determined, the TDR waveform can be obtained by standard spectral analysis that involves (1) transforming the incident step input into the frequency domain; (2) determining the frequency response of the output using equations (8), (10), and (11), and (3) transforming the frequency response back into the time domain. The Fourier and inverse Fourier transforms were performed using the fast Fourier transform (FFT) and the inverse FFT (IFFT) algorithm. Appropriate zero padding and a suitable window size for frequency and time were used to prevent aliasing in the discrete Fourier transform.

[17] The transmission line parameters of the TDR probe must be known when comparing the measured waveform with the simulated waveform. The transmission line of the TDR measurement system was divided into four parts: (1) front panel, (2) coaxial cable, (3) probe head, and (4) TDR probe, as illustrated in Figure 5. The line parameters were calibrated using a model-based inverse analysis. The dielectric permittivity, the reference characteristic impedance, and the length of each uniform section characterize a nonuniform transmission line. Various combinations of reference impedance and dielectric permittivity can result in identical impedance, while different combinations of section lengths and dielectric constants can yield the same propagation delay (i.e., $\gamma_{i} l_{i}$ ). Consequently, this set of three parameters has only two degrees of freedom. Considering these three line parameters in calibration would lead toward nonuniqueness. However, material with known dielectric properties was used to calibrate the probe section. Obtaining the correct waveform requires only that the front panel, coaxial cable, and probe head have equivalent line parameters, such that they have the same impedance and propagation delay. Hence a value of one of the three parameters for the front panel, cable, and the probe head, was assumed. The remaining two parameters were back- calculated from the measured waveform in a material with known dielectric permittivity.

\subsection{Layer-Peeling Algorithm}

[18] Only the properties of the material inside the probe remain unknown and are of interest after the transmission line parameters are calibrated. The probe system and the tested material together determine the TDR waveform. Accordingly, the waveform contains information on both the system parameters and the material properties. Preprocessing the measured waveform to remove multiple reflections unrelated to the material under test helps in interpreting the data. After preprocessing, the waveform should look as if the probe (sample section) were directly connected to the TDR device, as illustrated in Figure 5. The reduced theoretical scatter function, $S_{11}^{\prime}$, of such a hypothetical ideal system is simpler than that of the overall TDR probe system. It can be written as

$$
S_{11}^{\prime}\left(\varepsilon_{r}^{*}(f), f\right)=\frac{Z_{\text {in }}\left(z_{n-1}\right)-Z_{S}}{Z_{\text {in }}\left(z_{n-1}\right)+Z_{S}}
$$

[19] The overall scatter function $\left(S_{11}\right)$ can be measured experimentally from the TDR waveform $(V(0))$ and input waveform $\left(V_{i n}\right)$ using equation (10). A more complicated procedure, however, is needed to measure the reduced scatter function. First, the overall input impedance is calcu-

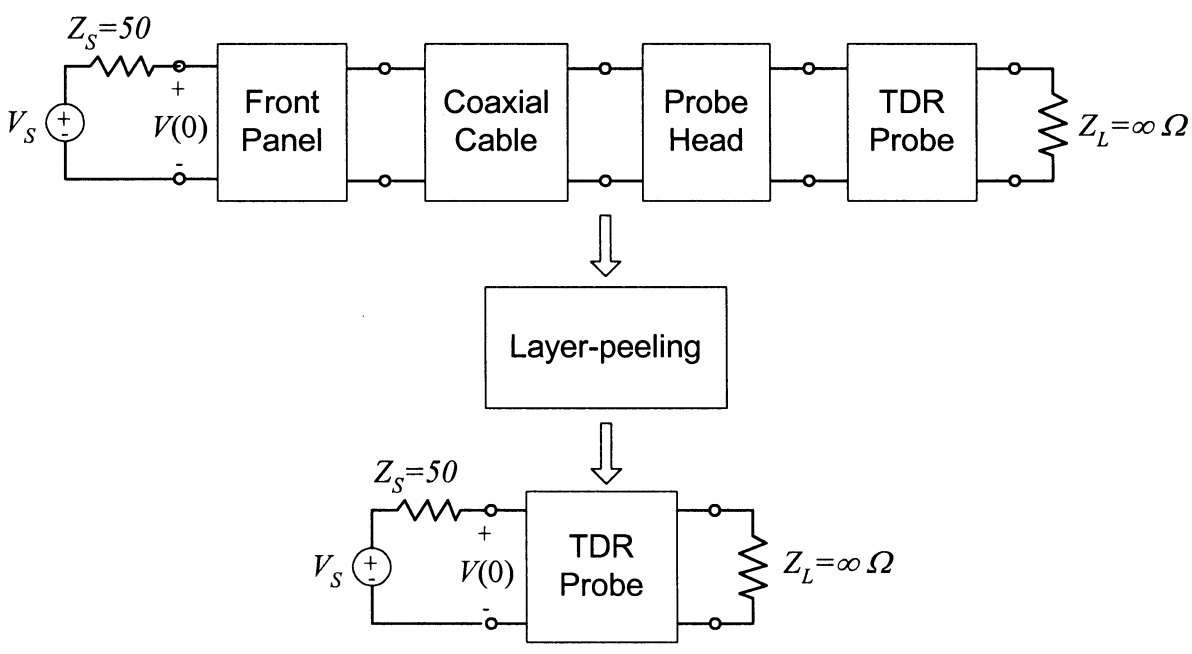

Figure 5. Schematic outline of the layer-peeling algorithm that reduces the nonuniform TDR system to an ideal probe system in which the probe is directly connected to the TDR device. 
lated from the measured $V(0)$ and $V_{i n}$ using equation (10) and (11) as

$$
\widetilde{Z}_{\text {in }}(0)=\frac{V(0)}{2 V_{\text {in }}-V(0)} Z_{S}
$$

where $\widetilde{Z}_{i n}(0)$ represents the "measured" overall input impedance. A layer-peeling algorithm formulated by reversing equation (8), the process for determining the overall input impedance, can be used to measure the input impedance looking into the probe section:

$$
\begin{gathered}
\widetilde{Z}_{i n}\left(z_{1}\right)=\frac{Z_{c, 1}\left[Z_{c, 1} \tanh \left(\gamma_{1} l_{1}\right)-\widetilde{Z}_{i n}(0)\right]}{\widetilde{Z}_{i n}(0) \tanh \left(\gamma_{1} l_{1}\right)-Z_{c, 1}} \\
\vdots \\
\widetilde{Z}_{i n}\left(z_{n-1}\right)=\frac{Z_{c, n-1}\left[Z_{c, n-1} \tanh \left(\gamma_{n-1} l_{n-1}\right)-\widetilde{Z}_{i n}\left(z_{n-2}\right)\right]}{\widetilde{Z}_{i n}\left(z_{n-2}\right) \tanh \left(\gamma_{n-1} l_{n-1}\right)-Z_{c, n-1}}
\end{gathered}
$$

[20] The input impedance looking into the sample section, $\tilde{Z}_{i n}\left(Z_{n-1}\right)$, obtained by the layer-peeling algorithm can then be used to measure the reduced scatter function and the TDR waveform of the hypothetical ideal probe system. The measured reduced scatter function $\tilde{S}_{11}^{\prime}$ becomes

$$
\widetilde{S}_{11}^{\prime}(f)=\frac{\widetilde{Z}_{i n}\left(z_{n-1}\right)-Z_{S}}{\widetilde{Z}_{i n}\left(z_{n-1}\right)+Z_{S}}
$$

[21] The scatter function of such a hypothetical ideal system is simpler than that of the overall TDR probe system, and the associated waveform includes only information concerning the material under test. The dielectric spectrum may be determined by setting equation (12) equal to equation (15) at each frequency. Alternatively, the dielectric spectrum may be parameterized using a dielectric dispersion model and back-calculated from the measured, reduced waveform or scatter function.

\section{Results and Discussion}

\subsection{Calibration of the Measurement System}

[22] The calibration of the transmission line parameters is a problem of inversion. The TDR system was divided into four parts, as shown in Figure 5. The line parameters of these parts were calibrated successively to reduce the number of unknowns in the inverse analysis. The measured waveforms of (1) the front panel terminated with a $50-\Omega$ impedance block, (2) the coaxial cable connected to the front panel, and (3) the deionized water in the TDR probe were used to calibrate the front panel, the coaxial cable, and the probing section, respectively. Initial values of the parameters to be inverted were assumed. Optimal values of the parameters were obtained by minimizing the residual sum of squares of the difference between the measured and simulated waveforms using the Simplex algorithm [Nelder and Mead, 1965].

[23] TDR waveforms show that 12 sections of uniform transmission lines of potentially different lengths and impedances should be able to describe the aberrations in the front panel, such that the front panel need not be divided into a lot of small sections of fixed equal length. The number of sections was determined by the number of reflections in the

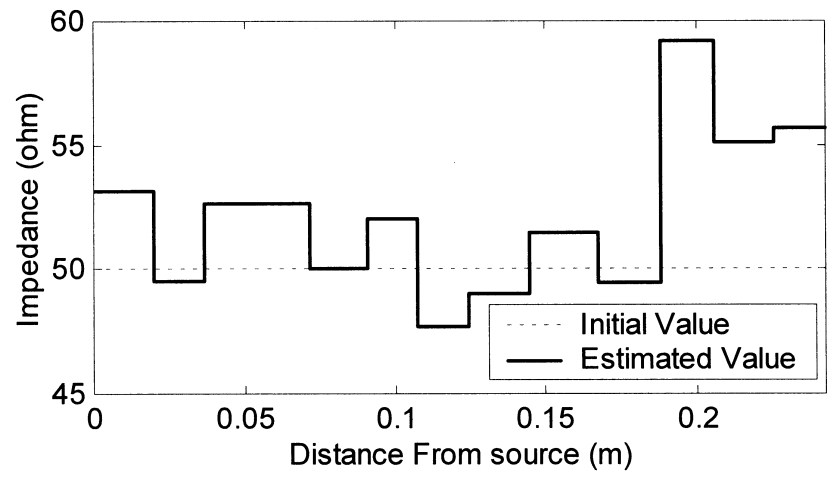

Figure 6. Calibrated impedance profile of the front panel aberration.

waveform shown in Figure 4. Assuming dielectric permittivity to be unity, 12 impedances and 12 lengths were inverted from the waveform measured by connecting the $50-\Omega$ terminating block to the front panel connector. The initial length of each section was set to $0.02 \mathrm{~m}$ and the initial impedance was set to $50 \Omega$. Figure 6 plots the estimated impedance profile following the inverse analysis. The predicted waveform matched the measured TDR input waveform quite closely, as indicated by the beginning of the waveforms in Figure 7. The coaxial cable has a nominal impedance of $50 \Omega$. However, the dielectric permittivity of the material inside the cable was modeled using the Debye equation to more accurately match the TDR waveform. The length of the cable was approximately measured and assumed to be $1 \mathrm{~m}$. The initial values of the reference impedance and Debye parameters were set to $\left(Z_{p}, \varepsilon_{s}, \varepsilon_{\infty}\right.$, $\left.f_{\text {rel }}, \sigma\right)=\left(72.5 \Omega, 2.2,2.1,1 \times 10^{8} \mathrm{~Hz}, 0 \mathrm{~S} / \mathrm{m}\right)$ and the estimated values were $\left(Z_{p}, \varepsilon_{s}, \varepsilon_{\infty}, f_{\text {rel }}, \sigma\right)=(71.24 \Omega, 2.56$, $\left.2.04,2.34 \times 10^{6} \mathrm{~Hz}, 0 \mathrm{~S} / \mathrm{m}\right)$. The TDR probe including the probe head was divided into three sections. For each section, one of the three line parameters was determined or assumed and the remaining two parameters were backcalculated from the TDR waveform measured in deionized water. Table 2 lists the initial and the estimated values of the line parameters. The waveforms predicted using the calibrated parameters match the measured waveforms closely, as indicated by the deionized water curve plotted in Figure 7. The system calibration was checked against measurements taken in materials with known dielectric properties. Figure 7 compares the simulated and measured TDR waveforms for tap water, butanol alcohol, and ethanol alcohol. The excellent match between simulated waveforms and measured waveforms further validates the wave propagation model and the system calibration.

\subsection{Layer-Peeling Algorithm}

[24] The calibrated system parameters and the dielectric properties of butanol were used to obtain the computersimulated TDR waveform. This simulated TDR waveform was then used to test the efficiency and accuracy of the layer-peeling algorithm. Figure 8 compares the reduced scatter function determined by the layer-peeling algorithm with that of the overall probe system. The signals that move back and forth in the transmission line are electromagnetic waves that establish a standing wave pattern. Its frequency of oscillation appearing in $S_{11}$ depends on the length of the 


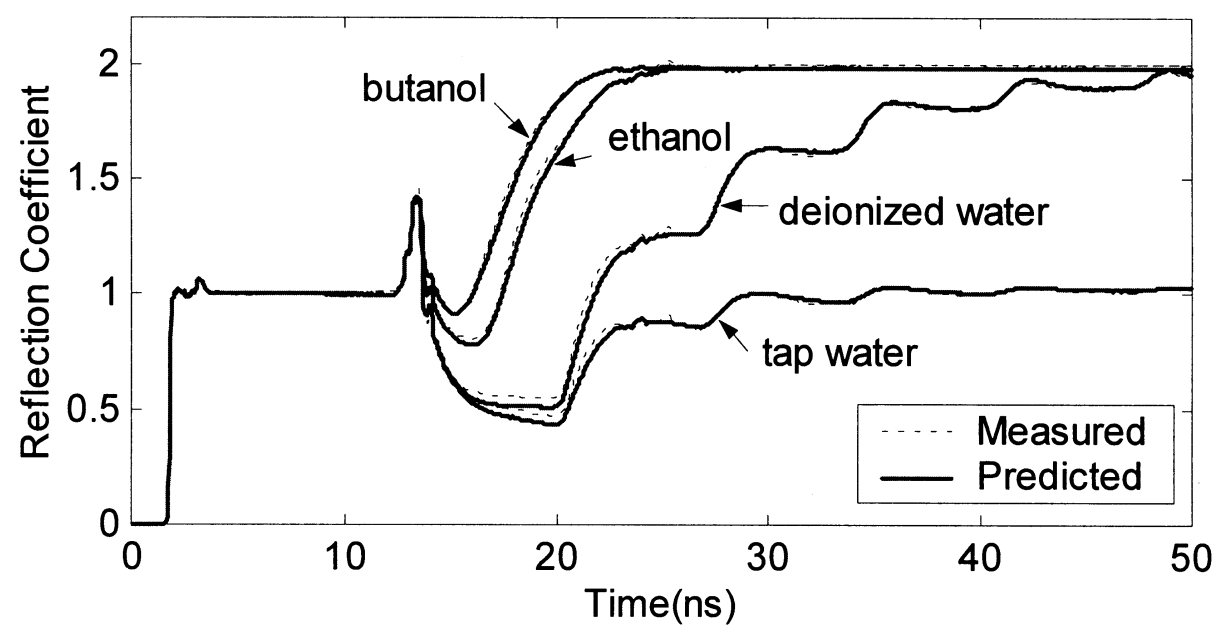

Figure 7. Comparison of the measured and predicted TDR waveforms in various liquids.

transmission line. The longer transmission line corresponds to a higher frequency of oscillation [Heimovaara et al., 1996]. Removing the long coaxial cable reduces the complexity of the scatter function considerably, as shown in Figure 8 . The layer-peeling process, as revealed by comparing the reduced scatter function with the directly simulated scatter function in Figure 8b, can remove the effect of the cable and the transitional device on the TDR measurement.

\subsection{Determining Dielectric Spectrum by Solving the Scatter Function}

[25] The only unknown in a TDR transmission line is the dielectric property of the material inside the probe after the system parameters of the TDR probe are appropriately calibrated. The dielectric spectrum may be determined by setting equation (12) equal to equation (15) at each frequency. The use of the reduced scatter function rather than the overall scatter function dramatically reduces the complexity of the nonlinear function, as shown in Figure 8. Notably, both data (i.e., the measured scatter function) and the unknown (i.e., the dielectric permittivity) are complex numbers. The unknown was represented as a $2 \times 1$ vector with the real and the imaginary part of dielectric permittivity, and the data were represented by a $2 \times 1$ vector with the amplitude and the phase angle of the scatter function. The nonlinear equation was solved by least squares optimization using the Simplex algorithm.

[26] The complexity of the nonlinearity of the scatter function as a function of dielectric permittivity increases with frequency. Figure 9 plots the error cost (i.e., the residual sum of squares) as a function of the real and imaginary parts of the dielectric permittivity at 18 and $360 \mathrm{MHz}$. The error cost is multimodal at high frequencies and the inverse solution is relatively sensitive to the initial value and errors in the data. The initial values of the dielectric permittivity at the two lowest frequencies were arbitrarily chosen as $40-1000 j$ in light of the well-posed inverse structure at low frequencies. However, the initial value of the dielectric permittivity for the following higherfrequency component was linearly extrapolated from the estimates of the preceding two frequencies to improve the convergence and stability of the inverse solution at high frequencies.

[27] The measured TDR waveform in butanol alcohol was preprocessed to obtain the reduced scatter function in the frequency domain. The dielectric spectrum was then determined from the equality between the measured and theoretical scatter function. Figure 10 plots the real part of the measured scatter function (denoted by "measured") and estimated dielectric spectrum (denoted by " $S_{11}$-fit"). The lowest frequency at which the dielectric spectrum can be estimated by solving the scatter function is $1 /\left(N^{*} \Delta t\right)$, where $N$ is the number of samples and $\Delta t$ is the sampling interval. In this study the waveforms were collected using $N=4096$ and $\Delta t=2.7 \times 10^{-11} \mathrm{~s}$, yielding a lowest frequency of $9 \mathrm{MHz}$.

[28] The results were compared with the theoretical scatter function and dielectric spectrum calculated using Debye parameters found in the literature [Heimovaara, 1994]. The difference between the measured and theoretical scatter functions is more significant at high frequencies, as shown in Figure 10a. This difference may be evidence of contribution of higher modes and fringing effect. Moreover,

Table 2. Initial and Estimated Values of Line Parameters Associated With the TDR Probe

\begin{tabular}{|c|c|c|c|c|c|c|c|c|c|c|}
\hline & \multicolumn{5}{|c|}{ Coaxial Head Section } & \multicolumn{3}{|c|}{ Air Gap Section } & \multicolumn{2}{|c|}{ Probe Section } \\
\hline & $Z_{p}, \Omega$ & $l,{ }^{\mathrm{a}} \mathrm{mm}$ & $\varepsilon_{s}$ & $\varepsilon_{\infty}$ & $f_{\text {rel }}, \mathrm{Hz}$ & $Z_{p}, \Omega$ & $l, \mathrm{~mm}$ & $\varepsilon^{\mathrm{a}}$ & $Z_{p}, \Omega$ & $l, \mathrm{~mm}$ \\
\hline Initial Value & 132 & 35.0 & 3.7 & 3.7 & $1 \times 10^{7}$ & 153 & 55.0 & 1 & 152.9 & 116.4 \\
\hline Estimated Value & 108.2 & 35.0 & 5.9 & 1.8 & $9.9 \times 10^{7}$ & 170.6 & 75.6 & 1 & 153.1 & 115.9 \\
\hline
\end{tabular}

\footnotetext{
${ }^{a}$ Assumed value.
} 

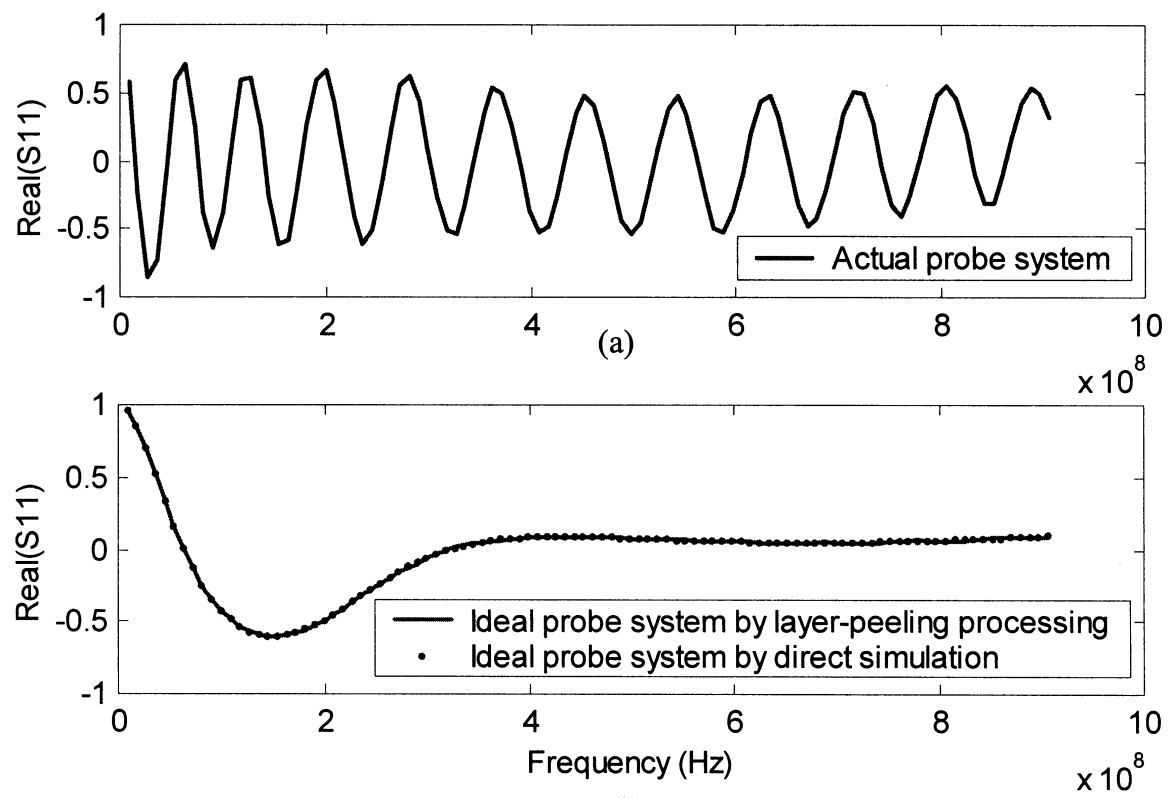

(b)

Figure 8. Real part of the scatter function (a) before and (b) after the layer-peeling processing.

the transmission line theory utilized here does not completely describe wave propagation at the interface at which the cross-sectional geometry changes dramatically. Consequently, the estimate of the dielectric spectrum gradually becomes less accurate at high frequencies due to the sensitivity to data error and the complexity of the scatter function at these frequencies. The accuracy is expected to improve if the cross-sectional dimension is reduced to increase the cutoff frequency of higher modes [Ramo et al., 1994] and if the magnitude of the source voltage is increased to enhance the signal-to-noise ratio. The design of the probe head should prevent abrupt changes in crosssectional geometry to minimize the inadequacy of wave propagation theory at high frequencies. Furthermore, a parametric study reveals that shortening the probe mitigates the complexity of the scatter function at all frequencies.
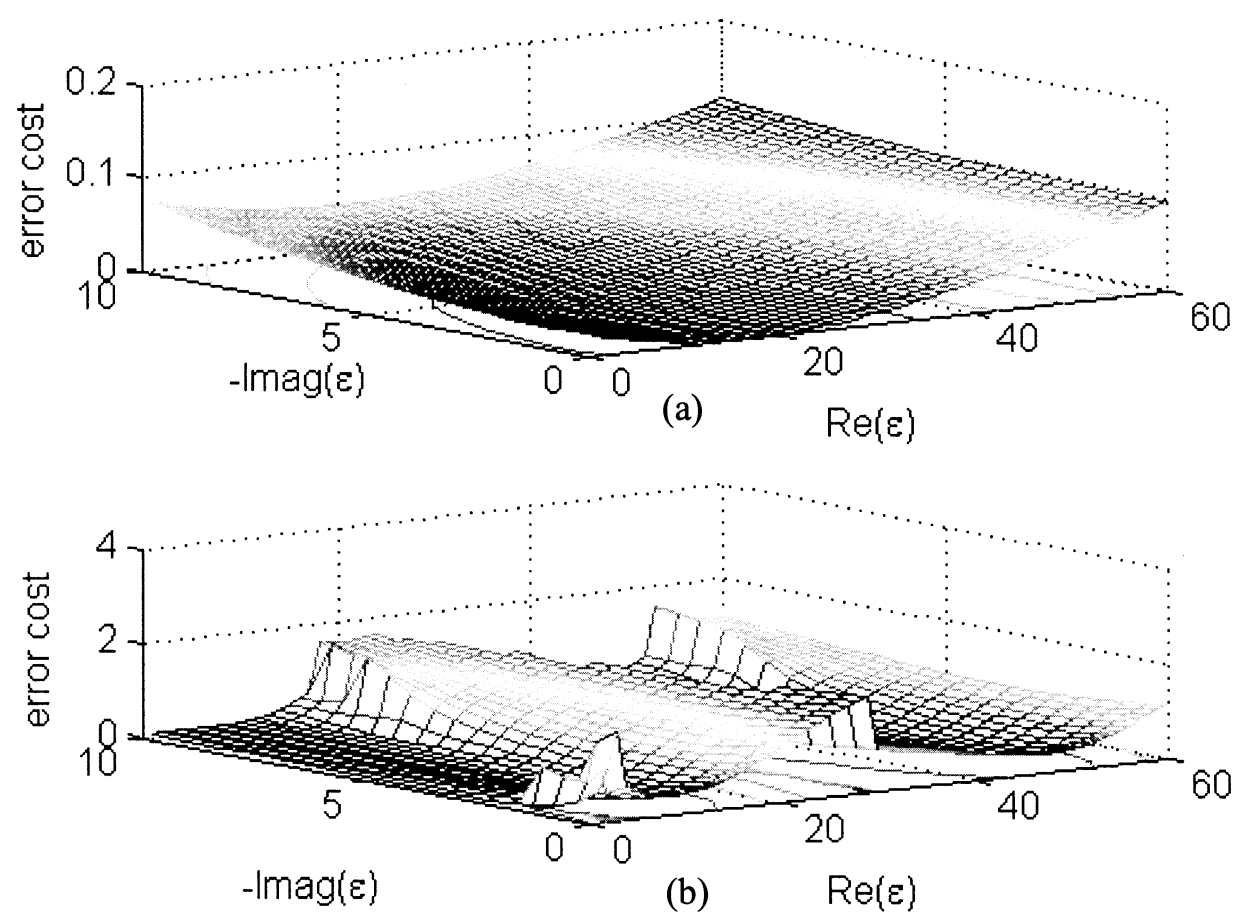

Figure 9. Error cost (residual sum of squares) as a function of real and imaginary parts of the dielectric permittivity at (a) $18 \mathrm{MHz}$ and (b) $360 \mathrm{MHz}$. The contours are shown on the imaginary $(\varepsilon)-\operatorname{real}(\varepsilon)$ plane. 

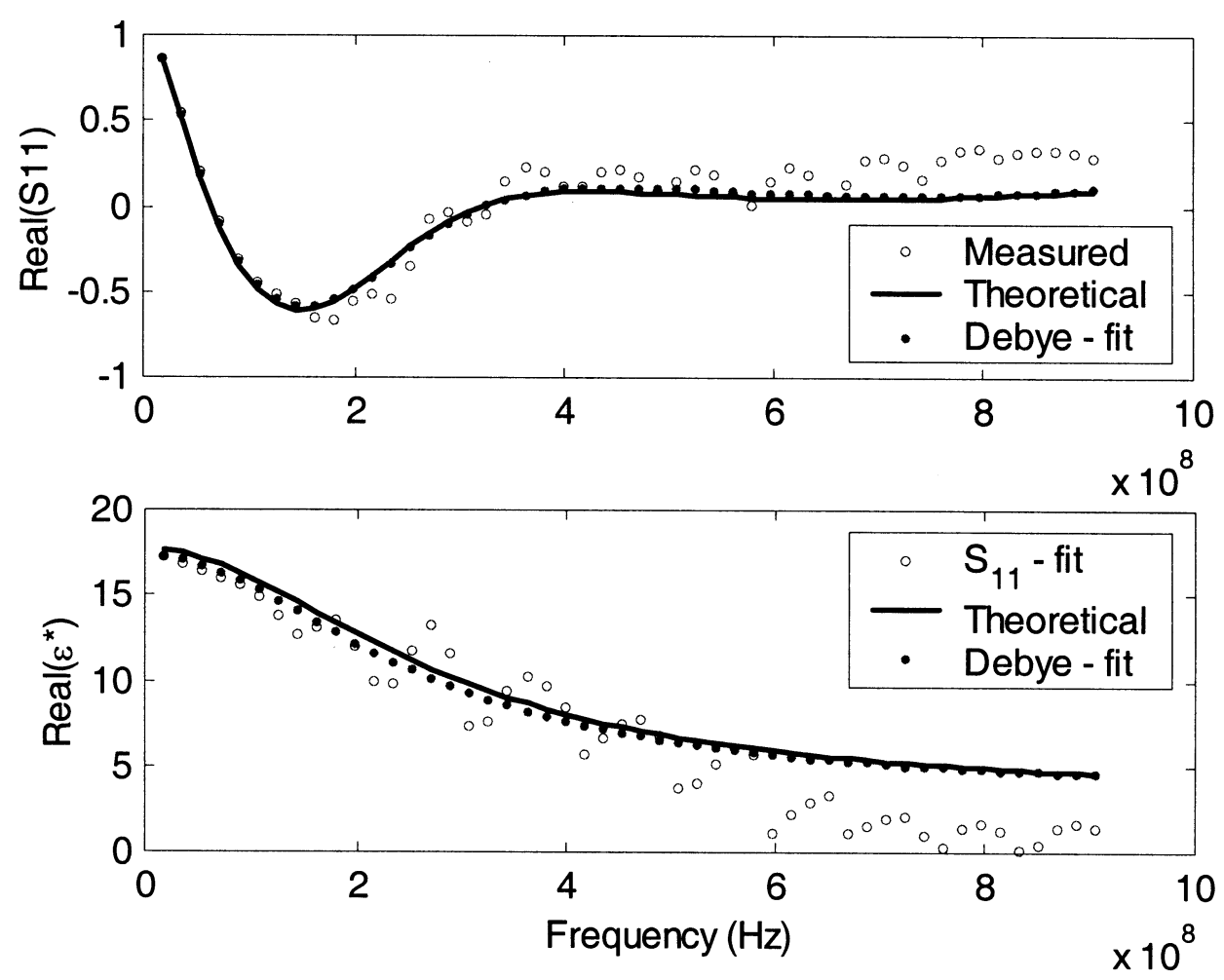

Figure 10. Estimated frequency-dependent dielectric properties of butanol alcohol.

Hence reducing the length of the probe further increases the stability of the inversion algorithm.

\subsection{Determining Dielectric Spectrum by Waveform Matching}

[29] The complex dielectric permittivity may be solved for directly using the scatter function at each frequency. However, the accuracy and precision of this method reduce as the frequency increases. Although the discrepancy between the measured and theoretical scatter functions at individual frequencies may be significant, the overall matches are reasonable. Consequently, the dielectric spectrum can feasibly be parameterized using a dispersion model such as the Debye equation or the volumetric mixing model, and the parameters can be calculated by fitting the measured scatter function or time domain waveform. Inversion was performed in the time domain because the TDR waveform contains real numbers rather than complex numbers. Both the original and reduced TDR waveforms obtained by the layer-peeling algorithm could be used for fitting. The latter is more efficient because the forward model involved in the inversion is evaluated using the ideal single-section system. The scatter function and dielectric spectrum estimated by waveform matching based on the Debye equation indicated by "Debye-fit" closely match the theoretical values, as shown in Figure 10. The Debye parameters $\left(\varepsilon_{s}, \varepsilon_{\infty}, f_{\text {rel }}, \sigma\right)$ were estimated as $(17.33,3.45$, $0.260 \mathrm{GHz}, 0.000015 \mathrm{~S} / \mathrm{m}$ ), and the theoretical values were $(17.7,3.3,0.274 \mathrm{GHz}, 0 \mathrm{~S} / \mathrm{m})$.

\subsection{Volumetric Mixing Model Versus Debye Equation}

[30] The accuracy of dielectric spectrum obtained using the waveform matching technique depends on the dielectric dispersion model. The dielectric property of a soil, as discussed above, can be parameterized by $\theta, \rho_{d}, A_{e s}, \sigma_{f w}$ $\sigma_{b w}$, and $\alpha$ in equation (7). These parameters can be backcalculated from the measured waveform in a fashion similar to that used in the method that is based on the Debye equation. Both the Debye equation and the volumetric mixing model have been used to model the dielectric relaxation of soils. Yet their applicability over various frequency ranges has not been compared. The dielectric spectrum of a silty loam was estimated by (1) solving the scatter function directly $\left(S_{11}\right.$-fit); (2) matching waveforms based on the Debye equation (Debye-fit), and (3) matching waveforms based on the volumetric mixing model (mixingfit). The optimal Debye parameters were $\left(\varepsilon_{s}, \varepsilon_{\infty}, f_{\text {rel }}, \sigma\right)=$ $\left(24.8,15.02,3.57 \times 10^{7} \mathrm{~Hz}, 0.0617 \mathrm{~S} / \mathrm{m}\right)$, while the optimal volumetric mixing parameters were $\left(\theta, \rho_{d}, A_{e s}, \sigma_{f w}, \sigma_{b w}\right.$, and $\alpha)=\left(0.1660,2.285 \mathrm{~g} / \mathrm{cm}^{3}, 175 \mathrm{~m}^{2} / \mathrm{g}, 0.0944 \mathrm{~S} / \mathrm{m}, 15.7400\right.$ $\mathrm{S} / \mathrm{m}, 0.6882$ ). Figure 11 shows the dielectric spectra determined by all three approaches. Similar to the result for butanol alcohol, the dielectric spectrum determined by solving the scatter function for each frequency separately appeared to be suspect above $200 \mathrm{MHz}$ due to the data error at high frequencies. However, this result still provides reference data that can be compared with the results obtained using a dielectric model, since the dielectric property of the soil is not known a priori. Comparing the measured waveform with that predicted by the back-calculated dielectric spectrum determines the data misfits of these three approaches. The waveform predicted by the $S_{11}$-fit method precisely matches the measured waveform because the dielectric permittivity is back-calculated independently at each frequency and no dielectric dispersion model is presumed. The small residual sum of squares (RSS = 

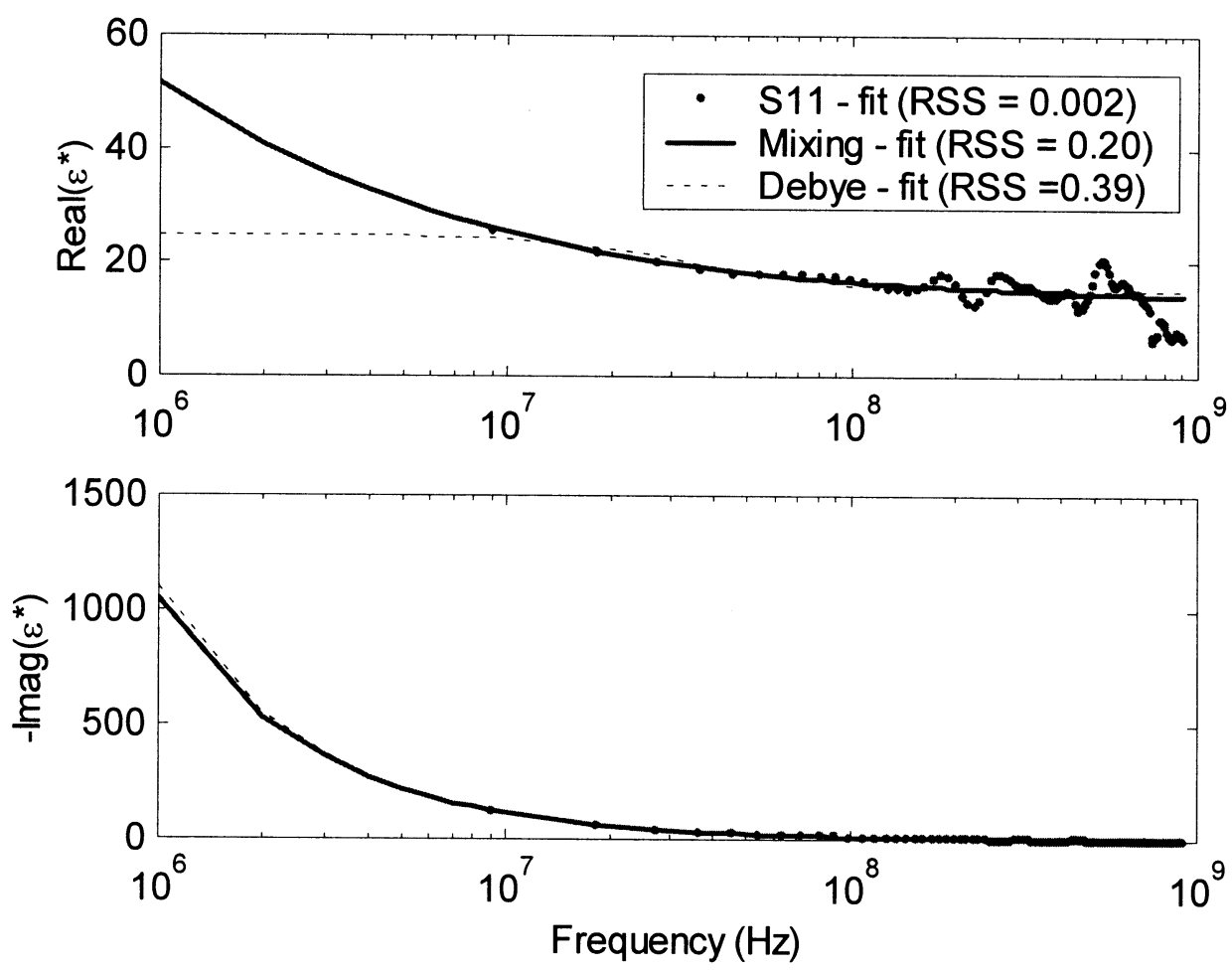

Figure 11. Estimated frequency-dependent dielectric properties of a silty loam.

0.002), shown in Figure 11, is due to numerical errors. Both model-based methods also have small RSS values, but the RSS of the Debye-fit method is double that of the mixing-fit method. Therefore both dielectric dispersion models are observed from Figure 11 to be capable of matching data estimated by solving the scatter function above $10 \mathrm{MHz}$, but the volumetric mixing model matched the data better at most frequencies. The main difference between the volumetric mixing model and the Debye equation is in how they predict the dielectric spectrum at low frequencies at which no data were available, by solving the scatter function. The Debye equation asymptotically approaches the static value as described by its functional form of equation (6) while the dielectric permittivity increases as the frequency decreases in the volumetric mixing model.

[31] The literature contains a substantial amount of information about the dielectric properties of soils up to a frequency of $10^{8} \mathrm{~Hz}$. Very high dielectric constants $\left(>10^{2}\right)$ have been measured at frequencies below $10^{4} \mathrm{~Hz}$ [Hoekstra and Delaney, 1974]. At low frequencies the dielectric permittivity is inversely proportional to frequency and levels off at a frequency of around $10^{7} \mathrm{~Hz}$. The functional constraint of the Debye equation is such that the equation cannot model the increase in dielectric permittivity at low frequencies, as shown in Figure 11. Therefore the volumetric mixing model is superior to the Debye equation in modeling the dielectric dispersion of soil over the entire range of frequencies. The Debye model was developed for aqueous materials. The contribution of the permanent dipole in the direction of the applied EM field is associated with thermal effects. However, water-bearing soils have a much broader distribution of relaxation times due to soil-water interactions that cannot be adequately modeled by the Debye model [Morgan and Lesmes, 1994; Lesmes and
Morgan, 2001]. The effective frequency range of TDR in this work is from $9 \mathrm{MHz}$ to $1.5 \mathrm{GHz}$. Although the Debye model can fit the dielectric dispersion curve over this frequency range, the model parameters are basically curve-fitting parameters that do not have precise physical interpretations. The volumetric mixing model, in contrast to the Debye model, has a physical interpretation and can model a broader distribution of relaxation times. However, the signal length must be considerably increased to enable the TDR system to measure the dielectric permittivity down to $10^{3} \mathrm{~Hz}$.

[32] The volumetric water content and bulk dry density were measured by conventional methods as $\theta=0.2295$ and $\rho_{d}=1.736 \mathrm{~g} / \mathrm{cm}^{3}$, respectively. These results differed markedly from the estimates of water content and density obtained from matching the TDR waveform using the sixparameter mixing model. If the water content and density were fixed using the conventionally measured values, the remaining four parameters could be inverted from the measured waveform as $\left(A_{e s}, \sigma_{f w}, \sigma_{b w}\right.$, and $\left.\alpha\right)=\left(348 \mathrm{~m}^{2} / \mathrm{g}\right.$, $0.3183 \mathrm{~S} / \mathrm{m}, 7.1096 \mathrm{~S} / \mathrm{m}, 0.5899)$. The volumetric mixing parameters thus obtained differed from the estimated values obtained using the six-parameter mixing model. However, the two resulting dielectric spectra were almost identical within the TDR bandwidth, implying that the volumetric mixing model can describe the dielectric relaxation spectrum for each individual soil specimen, but the six model parameters are correlated.

\section{Conclusions}

[33] The transmission line in a TDR measurement system is generally nonuniform, and the dielectric permittivity of the material may depend on frequency. Spectral analysis and 
input impedance were used to develop a wave propagation model that accounts for the dielectric dispersion of the material and the nonuniformity of the transmission line. The proposed numerical procedure is a relatively simple and powerful tool for probe design, parametric studies, data interpretation, and inverse analyses. The dielectric permittivity, reference characteristic impedance, and the length of each uniform section characterize a nonuniform transmission line. Inverting these three parameters necessarily causes nonuniqueness, but uniqueness can be achieved when one of the parameters is known or equivalently assumed. The TDR probe was calibrated from the TDR waveform measured in deionized water. The excellent match between the simulated and recorded waveforms validates the model.

[34] A layer-peeling algorithm was presented based on the new formulation of wave propagation. The effect of the cable and the probe head on the measurement system was examined and removed by applying the layer-peeling algorithm. Consequently, a relatively simple and robust measurement system could be used to measure the dielectric spectrum based on the nonuniform and dispersive model and the layer-peeling algorithm. One approach to measuring dielectric relaxation spectrum involves solving for the dielectric permittivity from the measured scatter function at each frequency separately. The rise time of the step pulse and the data length of the TDR waveform used in this study limited the measurement to an effective frequency range from $9 \mathrm{MHz}$ to $1.5 \mathrm{GHz}$. However, the data error and complexity of the scatter function increases with frequency such that the accuracy and precision decrease as the frequency increases. A reliable result could only be obtained below $200 \mathrm{MHz}$. Reducing the size of the probe and avoiding an abrupt change in the cross-sectional geometry inside the probe head may increase the accuracy at high frequencies.

[35] Alternatively, the dielectric spectrum can be paramterized using a dispersion model such as the Debye equation or the volumetric mixing model. Fitting the measured time domain waveform by adjusting the model parameters can determine the values of these parameters. This method is better than directly solving the scatter function at each frequency if an appropriate dielectric dispersion model is used. The dielectric spectrum of a soil was estimated by solving the scatter function directly, matching waveforms using the Debye equation, and matching waveform using the volumetric mixing model. The results showed that the volumetric mixing model was better than the Debye equation in describing the dielectric dispersion of a soil and yielding a dielectric spectrum that corresponded to that obtained by solving the scatter function. However, the six parameters of the volumetric mixing model are correlated, resulting in nonunique estimates. More work must be undertaken to elucidate the effect of these parameters on soil dielectric spectrum and perhaps simplify the parameterization of volumetric mixing models. Research is also under way to study layered soils using the wave propagation model and layer-peeling algorithm.

[36] Acknowledgments. The author gratefully acknowledges Vincent Drnevich, Richard Deschamps, and An-Bin Huang for reading an earlier draft. The suggestions of anonymous reviewers improved the paper.

\section{References}

Dobson, M. C., F. T. Ulaby, M. T. Hallikainen, and M. A. El-Rayes, Microwave dielectric behavior of wet soil, part ii, Dielectric mixing models, IEEE Trans. Geosci. Remote Sens., 23, 35-46, 1985.

Feng, W., C. P. Lin, R. J. Deschamps, and V. P. Drnevich, Theoretical model of a multisection time domain reflectometry measurement system, Water Resour. Res., 35, 2321-2331, 1999.

Friel, R., and D. Or, Frequency analysis of time-domain reflectometry (tdr) with application to dielectric spectroscopy of soil constituents, Geophysics, 64, 707-718, 1999.

Giese, K., and R. Tiemann, Determination of the complex permittivity from thin-sample time domain reflectometry, Adv. Mol. Relax. Processes, 7, 45-59, 1975.

Hasted, J. B., Aqueous Dielectric, Chapman and Hall, New York, 1973

Heimovaara, T. J., Frequency domain analysis of time domain reflectometry waveforms, 1, Measurement of the complex dielectric permittivity of soils, Water Resour. Res., 30, 189-199, 1994.

Heimovaara, T. J., W. Bouten, and J. M. Verstraten, Frequency domain analysis of time domain reflectormetry waveforms, 2, A four-component complex dielectric mixing model for soils, Water Resour. Res., 30, $201-$ $209,1994$.

Heimovaara, T. J., E. J. G. de Winter, W. K. P. van Loon, and D. C. Esveld, Frequency-dependency dielectric permittivity from 0 to $1 \mathrm{GHz}$ : Time domain reflectometry measurements compared with frequency domain network analyzer measurements, Water Resour. Res., 32, 3603-3610, 1996.

Hilhorst, M. A., Dielectric characterization of soil, Ph.D. thesis, Wageningen Agric. Univ., Wageningen, Netherlands, 1998.

Hoekstra, P., and A. Delaney, Dielectric properties of soils at UHF and microwave frequencies, J. Geophys. Res., 79, 1699-1708, 1974.

Lesmes, D. P., and F. D. Morgan, Dielectric spectroscopy of sedimentary rocks, J. Geophys. Res., 106, 13,392-13,346, 2001

Magnusson, P. G., G. C. Alexander, and V. K. Tripathi, Transmission Lines and Wave Propagation, 3rd ed., CRC Press, Boca Raton, Fla., 1992.

Morgan, F. D., and D. P. Lesmes, Inversion for dielectric relaxation spectra, J. Chem. Phys., 100, 671-681, 1994.

Nelder, J. A., and R. Mead, A simplex method for function minimization, Comput. J., 7, 308-313, 1965.

Ramo, S., J. R. Whinnery, and T. van Duzer, Fields and Waves in Communication Electronics, 3rd ed., John Wiley, New York, 1994.

Yanuka, M., G. C. Topp, S. Zegelin, and W. D. Zebchuk, Multiple reflection and attenuation of time domain reflectometry pulses: Theoretical considerations for applications to soil and water, Water Resour. Res., 24, 939-944, 1988.

C.-P. Lin, Department of Civil Engineering, National Chiao Tung University, 1001 Ta-Hsueh Road, Hisnchu, Taiwan. (cplin@mail.nctu.edu. tw) 